\title{
Granular segregation in a thin drum rotating with periodic modulation
}

\author{
Richard D. P. East, Philippa McGuinness, Finn Box, and Tom Mullin* \\ Manchester Center for Nonlinear Dynamics, School of Physics and Astronomy, University of Manchester, \\ Manchester M13 9PL, United Kingdom \\ Iker Zuriguel $^{\dagger}$ \\ Departamento de Física, Facultad de Ciencias, Universidad de Navarra, 31080 Pamplona, Spain \\ (Received 9 December 2013; revised manuscript received 7 October 2014; published 24 November 2014)
}

\begin{abstract}
We present the results of an experimental investigation into the effects of a sinusoidal modulation of the rotation rate on the segregation patterns formed in thin drum of granular material. The modulation transforms the base pattern formed under steady conditions by splitting or merging the initial streaks. Specifically, the relation between the frequency of modulation and the rotation rate determines the number of streaks which develop from the base state. The results are in accord with those of Fiedor and Ottino [J. Fluid. Mech. 533, 223 (2005)], and we show that their ideas apply over a wide range of parameter space. Furthermore, we provide evidence that the observed relationship is maintained for filling fractions far from $50 \%$ and generalize the result in terms of the geometry of the granular deposit.
\end{abstract}

DOI: 10.1103/PhysRevE.90.052205

PACS number(s): 45.70.Mg, 47.57.Gc, 83.80.Fg

\section{INTRODUCTION}

When a granular mixture flows inside a rotating drum or tumbler, segregation of the constituent components can occur $[1,2]$. In long horizontal rotating drums the different particles typically separate into vertical bands that interact with each other [3-5]. An essential part of the formation of such vertical bands is the initial creation of a segregated core of grains which is formed in the radial direction of the drum [5-8]. Understanding the origin of this initial radial segregation is a key factor in achieving a better understanding of the complete segregation process. One way to isolate and explore radial segregation is to use a thin rotating drum where axial segregation is suppressed.

Radial segregation in thin rotating drums occurs, for example, when particles differ in density [9] or size. In the latter case, smaller particles group to form a central core, and larger ones accumulate in the outer wall region. As the partially filled drum rotates, avalanching takes place down the free surface, and inertial and percolation effects lead to the deposition of small particles beneath larger ones [10-12]. At low rotation rates, the central core of small particles evolves into a qualitatively different segregated pattern [13-15] where small particles are concentrated into streaks (sometimes referred to as petals or lobes). In our experiment this occurs below approximately $0.4 \mathrm{rad} / \mathrm{s}(4 \mathrm{rpm})$, and examples of these streaks are shown in Fig. 1. This type of segregation is related to the stripe formation induced by avalanching during the building of a pile $[16,17]$. Nevertheless, other mechanisms play important roles in the pattern formation in a rotating drum as the streaks can be much thicker than the flowing avalanche layer at the granular surface [18-20].

The pattern which develops also depends on the filling level of the drum. Geometrical aspects are crucial when the filling level of the drum is far from 50\% [15,21-24]. For the case of a

\footnotetext{
*tom@reynolds.ph.man.ac.uk

†iker@unav.es
}

half-filled rotating drum, however, aspects such as the diameter of the drum, the proportion of small particles in the sample, and the rotation frequency are all important in the pattern selection process [18]. The number of streaks in the pattern is determined by a competition between stripe formation (in the surface layer) and coarsening.

A feature of this segregation process is that an uphill wave of big particles travels from the tip of the streaks of small particles to the center of the drum. Streaks are stable if they are separated by an angular distance, which allows the uphill wave to reach the center of the drum and flatten the surface. Otherwise, mixing occurs and the streaks merge [20] in the form of streak coarsening as reported in Ref. [19]. In practice, the results reported in Ref. [18] reveal that for rotation frequencies $\omega$ of the order of $0.05 \mathrm{rad} / \mathrm{s}$ or higher, the time lapse between the passage of two consecutive streaks $(T)$ was independent of the frequency of rotation. This behavior implies an inverse proportionality between the number of streaks $\left(N_{b}\right)$ developed in the drum and the frequency of rotation: $N_{b} \propto 1 / \omega$. Moreover, for the granular mixture used in Ref. [18], it was empirically found that $T=0.46 r_{c}$, where $T$ was measured in seconds and $r_{c}$ (which is the radius of the initial core of small particles) was measured in centimeters.

More recently it has been shown that the pattern established in a rotating drum can be controlled by modulating the rotation speed. Fiedor and Ottino [25] demonstrated that imposing a sinusoidal modulation on the rotation speed leads to the formation of patterns with different numbers of streaks to those obtained with a constant speed. For the particular case of a half-filled drum, odd frequencies of modulation (measured as the number of cycles per rotation, $f_{E}$ ) suppress streak segregation. On the other hand, modulation frequencies with an even number of cycles per rotation lead to a well-defined pattern where, at least for the cases investigated in Ref. [25], the number of streaks $(N)$ was half the number of cycles per rotation:

$$
N=f_{E} / 2 .
$$




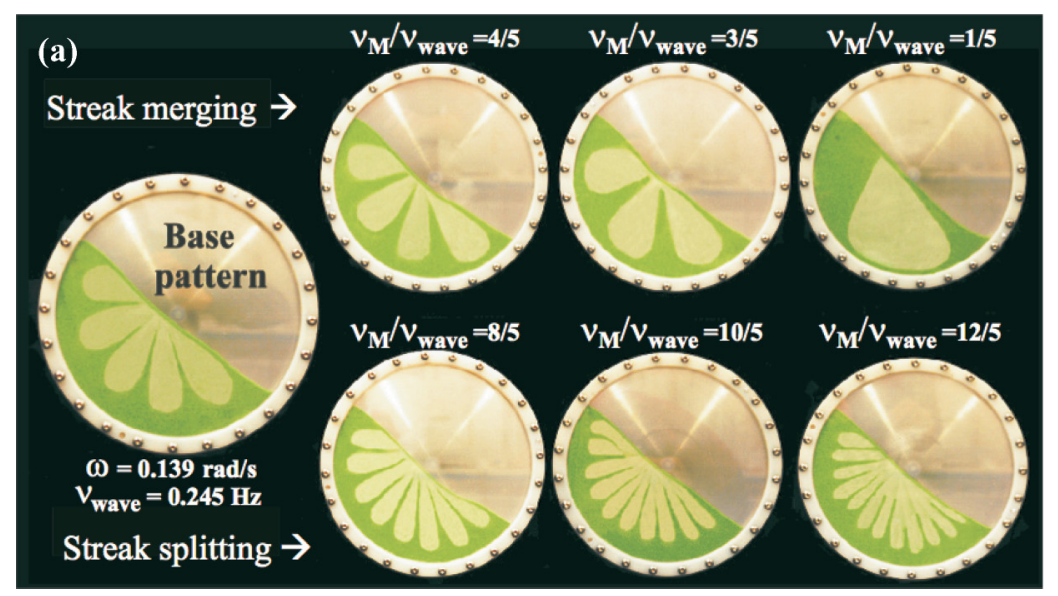

(b)

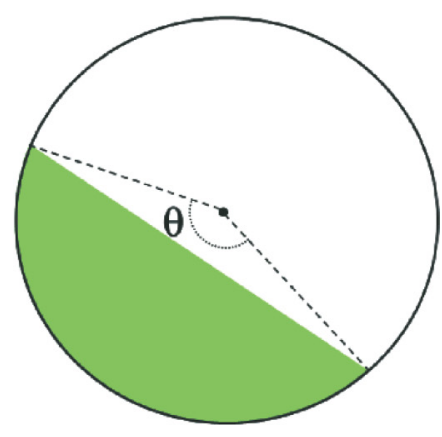

FIG. 1. (Color online) (a) Images of typical patterns developed when using a five-streak pattern (left figure) as the base state. At the top (bottom), patterns obtained after a streak-merging (splitting) process. In all these cases the frequency of rotation was $\omega=0.139 \mathrm{rad} / \mathrm{s}$, and the modulation frequency $v_{M}$ was varied as indicated in each figure. The definition of $v_{\text {wave }}$ is given in the main body text. (b) Schematic of the drum indicating $\theta$, the angular distance over which the streaks were arranged.

The explanation given for this behavior is that segregation takes place only during periods of acceleration in the flowing layer. This suggests the segregation pattern can be controlled by selecting an appropriate modulation frequency.

We explore the effect of the modulation of the rotation rate on the segregated pattern for different frequencies of modulation, filling fractions, amplitudes of modulation, and initial rotation rates of the drum. This was carried out by first obtaining a stable base pattern at a constant speed of rotation and then applying a modulation. The number of streaks developed before and after modulation was measured, and this enabled a direct comparison with the numerical and experimental work of Fiedor and Ottino [25]. The results obtained support for their calculations and observations and provide a generalization of their ideas over a wide range of rotation speeds, modulation frequencies, and filling fractions. In addition, we show that modulation may lead to either an increase or a reduction of the number of streaks. This demonstrates the modulation of rotation speed affects the final pattern through either streak splitting or streak merging.

\section{EXPERIMENTAL SETUP}

The experimental apparatus comprised a $3 \mathrm{~mm}$ thick drum of $D=24.5 \mathrm{~cm}$ diameter. The drum was machined from aluminium with a front wall of glass to allow direct observation of the patterns. The drum was mounted vertically and driven by a feedback-controlled DC motor connected to the drum via a belt drive attached to a gearbox. The supply to the motor was the sum of a steady voltage from a controlled DC supply and a sinusoidal modulation provided by a function generator. In an initial set of experiments the drum was half filled $( \pm 2 \%)$ with a mixture of glass particles of different sizes but equal density, as in Ref. [18]. The smaller white particles had a diameter of $120 \pm 30 \mu \mathrm{m}$, whereas the larger green particles had a diameter of $710 \pm 30 \mu \mathrm{m}$. The proportion of small particles gave a volume fraction of $\phi=0.35$ with respect to the large particles. A CCD camera was used to capture images, which were stored on an associated computer for further analysis.
A stable base pattern with a well-defined number of streaks was established by rotating the drum at a constant angular velocity $(\omega)$. In the first series of experiments, with the half-filled rotating drum, the values of $\omega$ used were $0.139 \mathrm{rad} / \mathrm{s}, 0.131 \mathrm{rad} / \mathrm{s}$, and $0.095 \mathrm{rad} / \mathrm{s}$, which resulted in patterns with five, six, and eight streaks, respectively. Once the base pattern was established, the angular velocity of the drum was modulated with a sinusoidal frequency $v_{m}$ and an amplitude of $0.05 \mathrm{rad} / \mathrm{s}$. Several modulation frequencies were superimposed on each of the steady rotation rates and led to transformation of the base patterns.

\section{DEPENDENCE OF THE PATTERN ON THE MODULATION FREQUENCY}

The first experimental finding was that the pattern transformation was dictated by the ratio of the frequency of modulation $\left(v_{M}\right)$ to the frequency of the granular wave $\left(v_{\text {wave }}\right)$, which travels from the tip of the streaks to the center of the drum. If the ratio $v_{M} / v_{\text {wave }}$ multiplied by the number of streaks in the base pattern was a small integer, stable patterns developed with a number of streaks that precisely correspond to this integer. As this number was approached, the uniformity of the size and the distribution of the streaks increased. This finding is further emphasized by the improvement in pattern definition which results for $v_{M} / v_{\text {wave }}=1$ although splitting or merging of streaks does not occur in this case. On the contrary, if the ratio $v_{M} / v_{\text {wave }}$ multiplied by the number of streaks in the base pattern was far from a small integer, a unstable configuration developed (i.e., patterns were not well defined) since they correspond to states with noninteger numbers of streaks and hence were incompatible with the boundary conditions.

In summary, once stable patterns were developed, $v_{M} / v_{\text {wave }}$ determined the final number of petals as $v_{M} / \nu_{\text {wave }}=N / N_{b}$, where $N$ is the number of petals in the final state and $N_{b}$ is the number of petals in the base state. For all base states, a ratio of $v_{M} / v_{\text {wave }}>1$ resulted in streak splitting while $v_{M} / \nu_{\text {wave }}<1$ led to streak merging. In Fig. 1 we present images of the patterns which developed after a modulation frequency was 


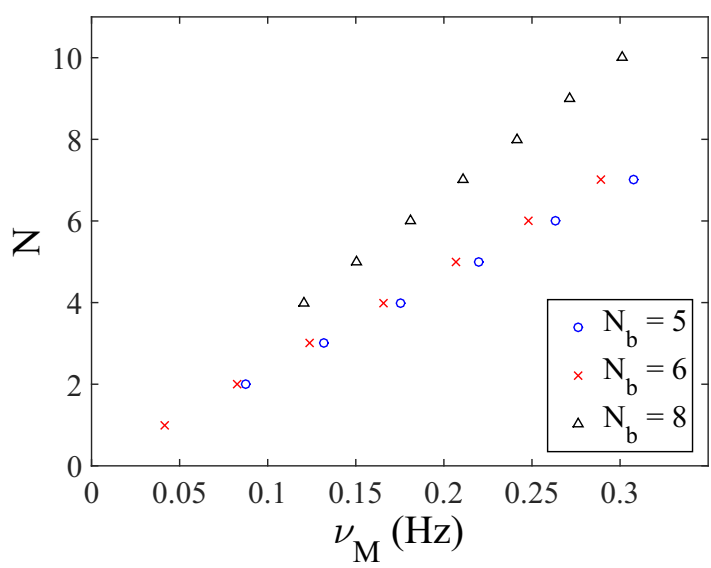

FIG. 2. (Color online) Number of streaks $(N)$ developed for different modulation frequencies $\left(v_{M}\right)$. Results, obtained with a half-filled drum, are presented for base patterns with different number of streaks $N_{b}$ (and hence different $\omega$ ) as indicated in the legend.

applied to a base pattern of five streaks formed with $\omega=$ $0.139 \mathrm{rad} / \mathrm{s}$ where $N / N_{b}$ is determined by $v_{M} / v_{\text {wave. This }}$ finding will be discussed below using ideas proposed by Fiedor and Ottino [22].

In Fig. 2 results are presented for the number of streaks developed after applying the modulation frequency to several initial base patterns. As shown in Fig. 1, increasing the value of the modulation frequency leads to a pattern with a larger number of streaks. The correlation is linear with a slope that depends on the initial number of streaks in the base pattern $\left(N_{b}\right)$ : the higher $N_{b}$, the greater the proportionality constant is between $N$ and $v_{M}$. This suggests the number of streaks in the base pattern is a scaling factor.

As explained above, in a drum rotated at constant speed, the time lapse $(T)$ between the passage of two streaks is approximately constant so that the number of streaks $\left(N_{b}\right)$ is inversely proportional to the rotation frequency $(\omega)$ [18]. Hence, an appropriate way to collapse the data is to plot $N \omega$ versus $v_{M}$, as shown in Fig. 3. The best linear fit applied to all the data is $N \omega=(3.16 \pm 0.08) v_{M}$. This result is analogous to the relationship given in Eq. (1) [25], which states that the number of streaks developed is half the number of cycles per rotation $\left(f_{E}\right)$. Indeed, $f_{E}$ can be written in terms of both the modulation frequency $\left(v_{M}\right)$ and the constant rotation frequency $(\omega)$ as $f_{E}=2 \pi v_{M} / \omega$. Substituting this for $f_{E}$ in Eq. (1) gives $N=\pi v_{M} / \omega$, which implies a correlation,

$$
N \omega=\pi v_{M},
$$

similar to the one displayed by the data in Fig. 3. It should be noted that unstable patterns were obtained for combined values of $v_{M}$ and $\omega$ that imply noninteger values of $N$. This observation is equivalent to the one made by Ref. [25] that odd values of $f_{E}$ produced poorly defined patterns. The result that $v_{M} / v_{\text {wave }}=N / N_{b}$ can be also deduced from Eq. (2). To this end, it should be appreciated that $v_{\text {wave }}$ is approximately the inverse of the time lapse between the passage of two consecutive streaks: $v_{\text {wave }}=1 / T$. Then, the constant frequency of rotation of the drum $(\omega)$ can be related with the frequency of the granular wave $\left(v_{\text {wave }}\right), \frac{\pi}{\omega}=\frac{N_{b}}{\nu_{\text {wave }}}$

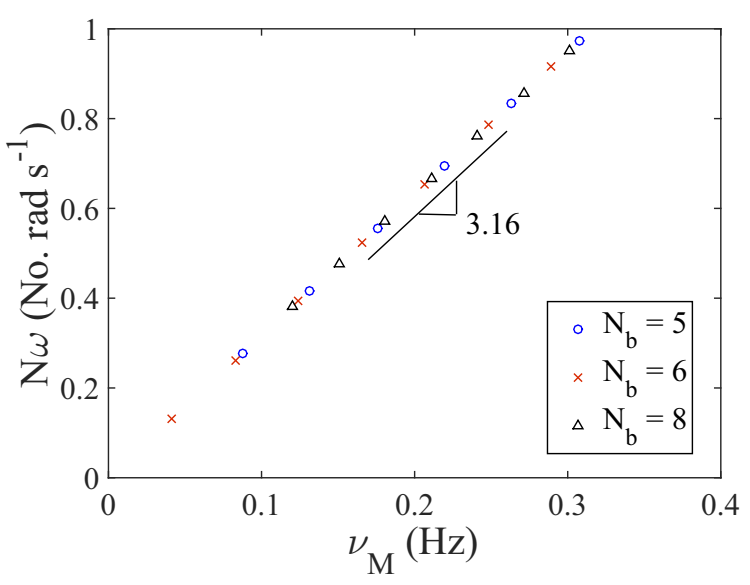

FIG. 3. (Color online) Number of streaks multiplied by the constant rotation frequency $(\omega)$ for different modulation frequencies and a filling fraction of 50\%. A linear fit of the results gives a relationship $N \omega=(3.16 \pm 0.08) v_{M}$. As in Fig. 2, we present results obtained with a half-filled drum with different number of streaks in the base pattern $N_{b}$ (and hence different $\omega$ ) as indicated in the legend.

where both sides of the equality account for the time taken by the drum to perform half a rotation. Replacing $\omega=v_{\text {wave }} \frac{\pi}{N_{b}}$ in Eq. (2), we obtain $v_{M} / v_{\text {wave }}=N / N_{b}$.

\section{ROLE OF FILLING FRACTION}

The dependence of the pattern transformation on $\omega$ and $v_{M}$ was extended over a wide range of filling fractions $\left(f_{f}\right)$ using the experimental method detailed above for a half-filled drum and the results are shown in Fig. 4. For each of the filling fractions, the resulting number of streaks was documented for various base states subjected to different frequencies of modulation. As in the case of the half-filled drum, filling fractions in the range 5\%-53\% exhibited a linear dependence of $N \omega$ on $v_{M}$ (Fig. 5). Interestingly, filling fractions different from $50 \%$ gave rise to constants of proportionality between $N \omega$ and $v_{M}$ different from $\pi$ found for $f_{f}=50 \%$. From now on, we will define $\theta_{c}$ to be the proportionality ratios such that $N \omega=\theta_{c} v_{M}$.

In Fig. 6 the results of $\theta_{c}$ (which are displayed by circles) reveal a monotonic increase with $f_{f}$. Decrease of the filling fraction produced a corresponding reduction in the associated angle $(\theta)$ at which the particles were deposited, and this determined the arrangement of the streaks in the drum. These are the values indicated in Fig. 6 by squares, which were calculated as the angle between the top and bottom of the avalanching layer measured from the center of the drum [see the schematic diagram in Fig. 1(b)]. The equivalence between the measured angular distance over which the particles are distributed in the drum, $\theta$, and the constant of proportionality determined from the relation $N \omega=\theta_{c} v_{M}$ for different filling fractions is striking. Indeed, if we assume that the surface of the avalanche was flat, $\theta$ can be obtained analytically as a function of the filling fraction $\left(f_{f}\right)$ as follows. The filling fraction is related to the area of the circular segment occupied by the grains $A_{C S}$ [green surface in Fig. 1(b)] and the projected area of the drum $A_{D}$ as $f_{f}=100 \frac{A_{C S}}{A_{D}}$. The area of 


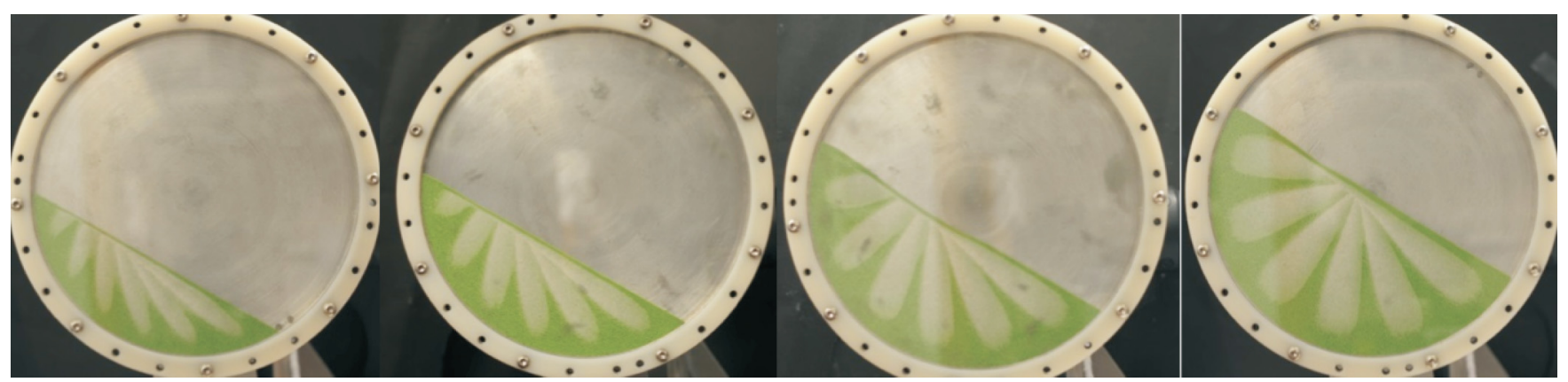

FIG. 4. (Color online) Six streak patterns for filling fractions of 20\%, 26\%, 39\%, and 50\% (left to right).

the circular segment is given by $A_{C S}=\frac{R^{2}}{2}[\theta-\sin (\theta)]$ and the projected area of the drum $A_{D}=\pi R^{2}$, where $R=D / 2$. This gives $f_{f}=100 \frac{[\theta-\sin (\theta)]}{2 \pi}$, which is indicated by the continuous line in Fig. 6.

In summary,

$$
N \omega=\theta v_{M}
$$

is a generalization of the previously result $N \omega=\pi v_{M}$ [Eq. (2)] for the half-filled rotating drum. This geometrical control of the pattern selection process can be understood recalling that segregation is produced in each acceleration phase of the drum [25]. Therefore, filling fractions below 50\% imply that in a complete rotation of the drum, the grains perform a number of periods of acceleration smaller than $N=\pi \nu_{M} / \omega$. Indeed, the number of periods of acceleration depends on the angle $\theta$, which is the proportionality constant of the generalized equation for different filling fractions.

Interestingly, varying the filling fraction displaced the center of the streaks from the center of the drum. As can be seen in Fig. 4, for $f_{f}<50 \%$ the center of the pattern moved to the left (uphill), and for $f_{f}>50 \%$ (not shown) the point

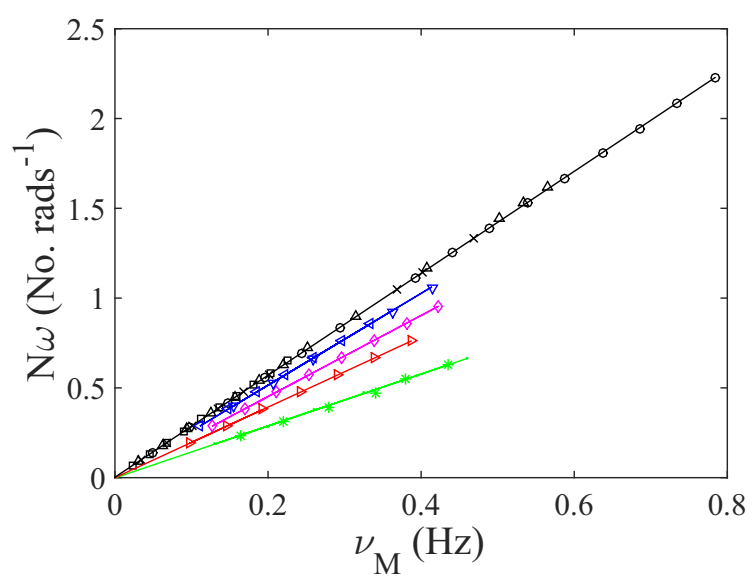

FIG. 5. (Color online) $N \omega$ measured as a function of $v_{M}$ for $f_{f}=$ $43 \%$ and $N_{b}=5(\mathrm{o}), 6(\mathrm{x}), 8(\triangle)$, and $10(\square) ; f_{f}=34.2 \%$ and $N_{b}=$ $5(\triangleleft)$ and $7(\nabla) ; f_{f}=25.7 \%$ and $N_{b}=6(\diamond) ; f_{f}=20 \%$ and $N_{b}=6$ $(\triangleright)$; and $f_{f}=10 \%$ subjected to an initial steady rotation of $\omega=0.04$ $(*)$ and $0.05 \mathrm{rad} / \mathrm{s}(\bullet)$. A linear fit of the form $N \omega=\theta_{c} v_{M}$ returns values of $\theta_{c}=2.86 \pm 0.06,2.57 \pm 0.08,2.23 \pm 0.06,1.97 \pm 0.04$, and $1.45 \pm 0.04$ for filling fractions of $43 \%, 34.2 \%, 25.7 \%, 20 \%$, and $10 \%$, respectively. moved to the right. The width and spacing of the streaks also decrease with decreasing filling fraction. Furthermore, for very small filling fractions $(\leqslant 10 \%)$, no streaks were discernible from the radially segregated initial core, even after $18 \mathrm{~h}$ of steady rotation. Application of a modulation of the angular frequency of rotation rapidly produced petal patterns even in the absence of a base pattern as shown in Fig. 7, and the familiar linear relationship between $N \omega$ and $v_{M}$ was uncovered, as shown in Fig. 5. Very high filling fractions $\left(f_{f}>55 \%\right)$ led to nonuniform base states, comprising asymmetrically distributed streaks of unequal size, and modulation of the angular frequency of the drum did not result in the splitting or merging of the streaks. Indeed, it was observed that, for a filling fraction of $58 \%$, the streaks in the base state were orientated tangentially to the locus of the streak center, similar to the pattern previously reported by Gray and Hutter [26] for a greater filling fraction.

\section{EFFECT OF THE AMPLITUDE OF MODULATION}

Finally, the role of the amplitude of the modulation on the splitting or merging process was investigated for various

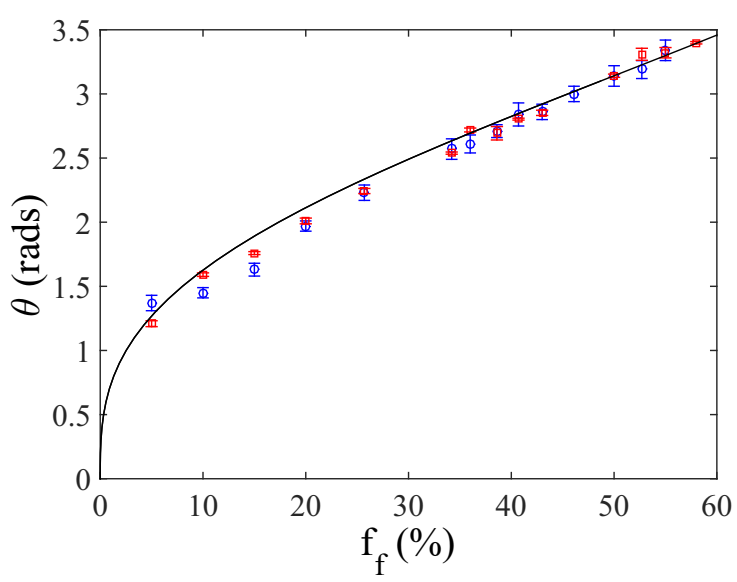

FIG. 6. (Color online) $\theta$ versus the filling fraction $\left(f_{f}\right)$. o represent the constants of proportionality, $\theta_{c}$, in the linear relationship $N \omega=\theta_{c} v_{M}$ determined from fits to the data collapse from various base states. $\square$ indicate the angular distance $\theta$ over which the particles deposit in the drum measured [as schematized in Fig. 1(b)] using image analysis. The solid line represents the analytical relation between $\theta$ and $f_{f}$ obtained assuming that the avalanching surface is flat. 


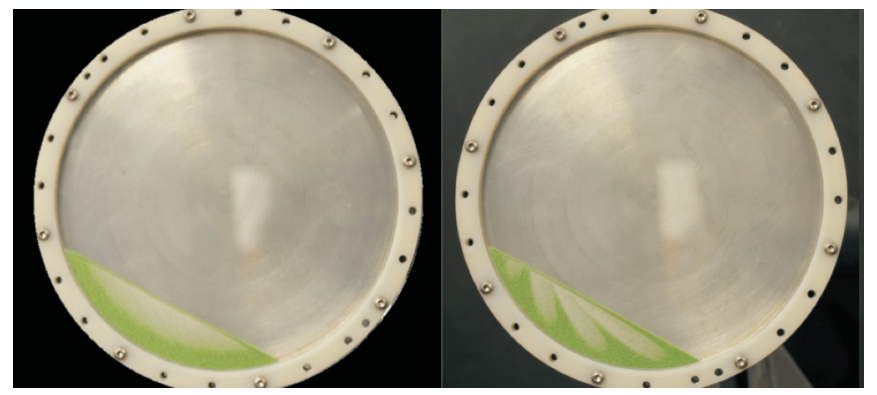

FIG. 7. (Color online) A filling fraction of $10 \%$ with steady rotation of $\omega=0.095 \mathrm{rad} / \mathrm{s}$ (left) and exhibiting four streaks after modulation with $v_{M}=0.0045 \mathrm{rad} / \mathrm{s}$ (right).

filling fractions. Decreasing the amplitude of modulation was found to increase the timescale of the pattern transformation. In Fig. 8 the time taken for the transition from a six-streak base state to a five-streak final state is shown as a function of the amplitude of modulation for filling fractions of $50 \%$ and $20 \%$. The results suggest that a finite amplitude is required for pattern transformation to occur and that the required amplitude scales with the filling fraction. Providing that the amplitude of modulation was sufficient for transformation, however, the number of streaks formed for a specific frequency of modulation was found to be independent of the amplitude of modulation.

\section{DISCUSSION}

We have shown that the number of streaks developed in a drum with a modulated rotation rate grows linearly with the frequency of modulation. The proportionality constant is determined by the mean value of the frequency of rotation of the drum and is equivalent to the angular distance over which the particles are deposited. This behavior is in accord with the findings of Fiedor and Ottino [25], which show that the number of streaks developed in a half-filled rotating drum is half the number of cycles per rotation. Importantly the expression reported here applies over a range of filling fractions $5 \%<f_{f}<55 \%$. This applies to both constant and modulation frequencies and is wider than had previously been appreciated.

Finally, we stress that our results contain an important implication for the understanding of radial streak segregation in rotating drums. Indeed, the reason argued in Ref. [25] to

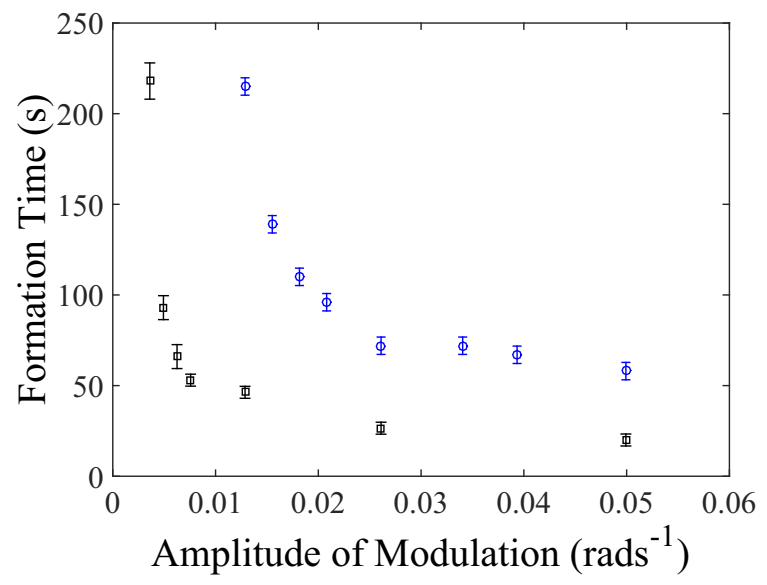

FIG. 8. (Color online) The time taken for a transition from six petals base pattern to five petals pattern measured as a function of the amplitude of the modulation for $f_{f}=50 \%$ (o) and $f_{f}=20 \%$ ( $\square$ ). The finite amplitude below which the transition was not observed to occur, even after time periods of $\sim 24 \mathrm{~h}$, can be seen to increase with increased filling fraction.

explain the effects of modulation was that streaks preferentially developed in each acceleration period. Here we explicitly show that each modulation (acceleration or deceleration) gives rise to streak formation. Moreover, streak merging occurs if the frequency of modulation is less than the frequency of the uphill granular wave. In other words, modulation is a mechanism of streak formation and an effective way of driving the splitting or merging of an existing pattern. This is in accord with Ref. [18], where two streaks were shown to merge if their angular distance was insufficient to allow the uphill wave to reach the center of the drum. Hence we propose that each modulation leads to a merging process during the acceleration phase and a splitting process during the deceleration phase. This conjecture is supported by the observation that the uphill wave reached the center of the drum during the slowest part of the rotation period.

\section{ACKNOWLEDGMENTS}

The experimental work was supported by two separate grants from the Ogden Trust. This work has been also financially supported by Project FIS2011-26675 MINECO (Spain).
[1] S. W. Meier, R. M. Lueptow, and J. M. Ottino, Adv. Phys. 56, 757 (2007).

[2] J. M. Ottino and D. V. Khakhar, Annu. Rev. Fluid Mech. 32, 55 (2000).

[3] O. Zik, D. Levine, S. G. Lipson, S. Shtrikman, and J. Stavans, Phys. Rev. Lett. 73, 644 (1994).

[4] I. Zuriguel, J. F. Boudet, Y. Amarouchene, and H. Kellay, Phys. Rev. Lett. 95, 258002 (2005).

[5] K. M. Hill, A. Caprihan, and J. Kakalios, Phys. Rev. Lett. 78, 50 (1997).
[6] N. Taberlet, W. Losert, and P. Richard, Europhys. Lett. 68, 522 (2004).

[7] Z. S. Khan, W. A. Tokaruk, and S. W. Morris, Europhys. Lett. 66, 212 (2004); Z. S. Khan and S. W. Morris, Phys. Rev. Lett. 94, 048002 (2005).

[8] M. Newey, J. Ozik, S. M. van der Meer, E. Ott, and W. Losert, Europhys. Lett. 66, 205 (2004).

[9] C. C. Liao, S. S. Hsiau, and H. C. Nien, Phys. Rev. E 89, 062204 (2014). 
[10] E. Clément, J. Rajchenbach, and J. Duran, Europhys. Lett. 30, 7 (1995).

[11] F. Cantelaube and D. Bideau, Europhys. Lett. 30, 133 (1995).

[12] G. G. Pereira and P. W. Cleary, Granular Matter 15, 705 (2013).

[13] D. V. Khakhar, A. V. Orpe, and J. M. Ottino, Powder Tech. 116, 232 (2001).

[14] D. V. Khakhar, A. V. Orpe, and S. K. Hajra, Physica A 318, 129 (2003).

[15] K. M. Hill, G. Gioia, and D. Amaravadi, Phys. Rev. Lett. 93, 224301 (2004); K. M. Hill, G. Gioia, D. Amaravadi, and C. Winter, Complexity 10, 79 (2005).

[16] J. C. Williams, Powder Tech. 2, 13 (1968).

[17] H. A. Makse, S. Havlin, P. R. King, and H. E. Stanley, Nature (London) 386, 379 (1997); H. A. Makse, P. Cizeau, and H. E. Stanley, Phys. Rev. Lett. 78, 3298 (1997); H. A. Makse, R. C. Ball, H. E. Stanley, and S. Warr, Phys. Rev. E 58, 3357 (1998).
[18] I. Zuriguel, J. M. N. T. Gray, J. Peixinho, and T. Mullin, Phys. Rev. E 73, 061302 (2006).

[19] S. W. Meier, D. A. M. Barreiro, J. M. Ottino, and R. M. Lueptow, Nat. Phys. 4, 244 (2008).

[20] I. Zuriguel, J. Peixinho, and T. Mullin, Phys. Rev. E 79, 051303 (2009).

[21] G. Metcalfe, T. Shinbrot, J. J. McCarthy, and J. M. Ottino, Nature (London) 374, 39 (2002).

[22] K. M. Hill, D. V. Khakhar, J. F. Gilchrist, J. J. McCarthy, and J. M. Ottino, Proc. Natl. Acad. Sci. USA 96, 11701 (1999).

[23] J. M. N. T. Gray and A. R. Thornton, Proc. R. Soc. A 461, 1447 (2005); A. R. Thornton, J. M. N. T. Gray and A. J. Hogg, J. Fluid Mech. 550, 1 (2006).

[24] K. M. Hill, N. Jain, and J. M. Ottino, Phys. Rev. E 64, 011302 (2001).

[25] S. J. Fiedor and J. M. Ottino, J. Fluid. Mech. 533, 223 (2005).

[26] J. M. N. T. Gray and K. Hutter, Continuum Mech. Thermodyn. 9, 341 (1997). 